\title{
Can knowledge of germline markers of toxicity optimize dosing and efficacy of cancer therapy?
}

\author{
Daniel Crona ${ }^{1}$ and Federico Innocenti $i^{1, *}$ \\ ${ }^{1}$ Institute for Pharmacogenomics and Individualized Therapy, University of North Carolina, Chapel \\ Hill, NC, USA 27599-7361
}

\section{Abstract}

The systemic treatment of cancer with traditional cytotoxic chemotherapeutic agents and more targeted agents is often complicated by the onset of adverse drug reactions. Pharmacogenetic prediction of adverse drug reactions might have consequences for dosing and efficacy. This review discusses relevant examples where the germline variant-toxicity relationship has been validated as an initial step in developing clinically useful pharmacogenetic markers and provides examples where germline variants have influenced dosing strategies and/or survival or other outcomes of efficacy. This review will also provide insight into the reasons why more pharmacogenetic markers have not been routinely integrated into clinical practice.

\section{Keywords}

5-fluorouracil; 6-mercaptopurine; bevacizumab; cetuximab; dosing; erlotinib; irinotecan; pharmacogenetics; survival; toxicity

It is well established that when cancer patients are administered chemotherapy, their clinical response will vary greatly [1-3]. While many patients experience a complete or partial response, for a sizable proportion of these patients the chemotherapy will be largely ineffective despite the onset of toxicities, which might be severe and even life threatening. Often, these adverse drug reactions (ADRs) will lead to hospitalizations [4]. Numerous factors influence whether a patient will experience an ADR, including baseline clinical and demographic characteristics and genetics. Among available efficacious regimens, the selection of the safest regimen for a given patient is not guided by molecular biomarkers, but rather by considerations related to the patient's age, comorbidities and performance status.

Traditional cytotoxic chemotherapy and more novel molecularly targeted agents (some of them are also called 'biologics') are the two core pharmacotherapeutic paradigms at the center of modern cancer care. Phase I trials identify the dose-limiting toxicities (DLTs) of a new chemotherapeutic agent, and doses are advanced until the onset of DLTs to define the agent's maximum tolerated dose (MTD) to be used in Phase II trials [5]. For most approved

\footnotetext{
(C) 2012 Future Medicine Ltd

*Author for correspondence: Tel.: +19199669422 innocent@unc.edu.

For reprint orders, please contact: reprints@futuremedicine.com

Financial \& competing interests disclosure

This work was supported, in part, by a National Institutes of Health grant (NIH/NCI K07CA140390-01) and also by an American Foundation for Pharmaceutical Education (AFPE) fellowship. F Innocenti receives royalties for the commercialization of the $U G T 1 A 1 * 28$ genetic test. The authors have no other relevant affiliations or financial involvement with any organization or entity with a financial interest in or a financial conflict with the subject matter or materials discussed in the manuscript apart from those disclosed. No writing assistance was utilized in the production of this manuscript.
} 
chemotherapeutic agents there is only one dose, rather than a dose range. This doseescalation design to discover the MTD intrinsically narrows the distance between toxic and effective doses. It is natural to expect that one single dose might not be tolerated by some patients and during the entire duration of the scheduled cycles of treatment. As a result, the onset of serious grade 3 or 4 toxicities (as defined by the National Cancer Institute Common Terminology Criteria for Adverse Events version 4.03 [201]) can largely affect drug dosing and dose intensity.

In all therapeutic areas, ADRs account for an estimated 7\% of all hospital admissions, occur in $10-20 \%$ of all patients and result in significantly increased hospital stays [6]. The costs to the health system associated with ADR-related morbidity exceed US $\$ 3.6$ billion annually in the USA. ADRs are consistently among the top ten reasons for death among patients [7]. Several studies have reported on the incidence of cancer chemotherapy related ADRs. A meta-analysis of 1219 colorectal cancer patients, who received 5-fluorouracil as part of their regimen, revealed that up to $31 \%$ experienced grade 3-4 neutropenia [8]. A more recent meta-analysis disclosed that approximately $30 \%$ of 2090 non-small-cell lung cancer (NSCLC) patients who received docetaxel experienced grade 3-4 neutropenia [9]. It is estimated that greater than 60,000 oncology patients are hospitalized due to severe neutropenia and its complications each year in the USA, and that neutropenia is associated with an inpatient mortality rate of $6.8 \%$ annually [6]. Dose-toxicity curves often differ between traditional cytotoxic chemotherapies and targeted molecular agents, and efficacy may occur in targeted agents at doses that do not cause significant toxicities [5]. Although the development of dosing strategies for targeted agents and biologics are still largely dependent on the identification of the MTD, there is significant debate concerning the practicality of abandoning traditional dosing strategies in favor of the identification of a minimal effective dose or the optimal biologic dose (OBD) [10]. OBD can be defined as the dose associated with a prespecified most desirable effect on a biomarker among all doses studied [5]. There are those who argue that the dosing of targeted agents should continue to follow the traditional MTD dose-finding model as they believe that there is still a lack of knowledge concerning OBD strategies [11]. However, others favor investigation of a minimal effective dose [12] or OBD [13,14].

Since dose-toxicity curves and OBD dosing strategies for targeted agents could be starkly different from traditional cytotoxic chemotherapy, the ADRs associated with these agents are rarely life-threatening, but still no less serious. These drugs tend to be associated with more nonhematological ADRs and less neutropenia than the traditional cytotoxics. ADRs, which adversely affect quality of life and activities of daily living, can result in noncompliance to oral targeted agents. For instance, a post approval survey of EGF receptor (EGFR) inhibitors revealed that $76 \%$ of respondents were noncompliant to therapy due to rash and that up to $32 \%$ of clinicians discontinued therapy due to rash [6]. Moreover, at least $11 \%$ of patients who receive cetuximab experience grade 3-4 rash [15], which could lead to dose modifications by the clinician or nonadherence by the patient, and ultimately complicate efficacy and survival outcomes. Up to $15 \%$ of patients treated with bevacizumab experience grade 3-4 hypertension [6] and are at a significantly higher risk of grade 3-4 proteinuria and hypertension at both low and high doses [16].

These common ADRs are still very concerning as medication nonadherence compromises dose intensity and ultimately survival [17,18]. Maintenance of dose intensity in patients for the entire course of their treatment is extremely important; it has been shown both prospectively and retrospectively, in a variety of tumor types, to be correlated with more positive outcomes [19,20]. Dose intensity is a function of both dose and frequency, and modifications to either chemotherapy dosing or frequency, as a result of ADRs, are detrimental to outcomes [21]. Dosing is often adjusted based on renal or hepatic function, 
hematologic counts, results of basic metabolic panels, or drug levels measured in plasma. In addition, chemotherapy package inserts also provide recommendations on how to adjust dosing (usually dose reductions or dose interruptions) based upon the onset of toxicity. Although very pragmatic, this approach of personalizing chemotherapy dose remains inefficient, expensive and adversely affects the quality of life of patients. However, certain ADRs have been researched as surrogate pharmacodynamic markers and seem to possess a predictive role for chemotherapy response and efficacy. The relationship between druginduced rash and survival has been investigated in EGFR inhibitors, such as erlotinib [2226]. Likewise, the relationships linking drug-induced hypertension, the VEGF-pathway inhibitor bevacizumab, and survival have been explored $[27,28]$. These studies support both the paradigm that these specific ADRs are likely pharmacodynamic proxies for efficacy, and the approach of dosing patients to OBD rather than MTD.

Pharmacogenetics examines interindividual genetic variability that influences the course of drug action so that medication regimens may be optimized to maximize response, while minimizing drug-induced toxicity. Personalized medicine through genetics and genomics is the overarching goal for the field of pharmacogenetics and vital to the evolution of effective treatment paradigms. The ability to predict and mitigate ADRs in oncology is of paramount importance as a majority of chemotherapeutic agents are dosed to their MTD and possess a narrow therapeutic index between efficacy and toxicity. There is currently a dearth of clinically relevant prognostic and predictive pharmacogenetic markers derived from germline variants that have been translated for routine incorporation into clinical practice [29-31]. There is an opportunity to better understand the relationship between germline variants, toxicity and chemotherapy dose so that more of these pharmacogenetic markers may be utilized clinically to individualize therapies for cancer patients.

The purpose of this review is to analyze the available literature to elucidate pertinent germline variant-ADR relationships and to describe how these associations may impact the delivery of an optimal chemotherapy dose to patients and consequently affect survival outcomes. We have chosen to systematically concentrate on a focused number of validated germline pharmacogenetic variants that associate with ADRs and the manner in which those variants might influence downstream effects, such as chemotherapy dosing and survival outcomes. This review should not be construed as a complete evaluation of all pharmacogenetic variants detailed in oncology-related literature, as this has been addressed extensively in many recent reviews [32-34]. However, the resulting effects of these associations on dosing and survival have never been analyzed in detail. For each specific gene discussed, we will not review a comprehensive list of all alleles in each of the genes. Rather, we have highlighted several specific pharmacogenetic variants that account for the majority of variability in each gene to emphasize our approach.

\section{Establishing the relationship between germline variants \& toxicity}

Validation of pharmacogenetic markers related to ADRs is of utmost importance to clinicians to allow them to personalize the most efficacious and safe regimen for each patient, without sacrificing dose intensity. With the advent of hypothesis-driven candidate gene studies and more unbiased genome-wide approaches, oncology researchers have begun to characterize the genetics that underlie serious ADRs. The labels for over 20 chemotherapeutic agents have been rewritten or revised to include information regarding pharmacogenetic markers. Specifically, seven of these agents (6-mercaptopurine, 6thioguanine, 5-fluorouracil, capecitabine, irinotecan, nilotinib and imatinib) include language describing a gene-toxicity relationship, which emphasizes the growing importance of validating clinically useful pharmacogenetic markers to prevent the onset or mitigate the severity of ADRs [202]. 
Numerous pharmacogenetic studies have been conducted in an attempt to identify novel germline variants that conclusively associate with ADRs. A majority of the validated pharmacogenetic markers, and those primarily discussed in this review, are germline variants that contribute to differences in drug metabolizing enzymes of drug inactivation. A classic example is the thiopurine antimetabolite 6-mercaptopurine, an integral component in the treatment of pediatric acute lymphoblastic leukemia (ALL) [35]. The antimetabolite, 6mercaptopurine, is activated in thioguanine nucleotides, which are then incorporated into the DNA and interfere with the activity of nucleic acid-processing enzymes [35]. TPMT inactivates 6-mercaptopurine through methylation. Genetic variations in the TPMT gene $(* 2, * 3 A$ and $* 3 C)$ lead to TPMT deficiency and reduced inactivation of 6-mercaptopurine [36], and patients carrying these mutations usually have increased thioguanine nucleotide erythrocyte levels [37,38].

The germline variant-toxicity relationship between UGT and irinotecan has also been studied extensively. UGT1A1 is responsible for the inactivation of the active metabolite of irinotecan through glucuronidation. Irinotecan is commonly used in advanced colorectal cancer in combination with 5-fluorouracil. An inverse relationship exists between the number of TA repeats in the UGT1A1 promoter and the transcriptional efficiency of the gene [39], its protein [40] and mRNA expression [41], and the level of glucuronidation of the active metabolite of irinotecan [42,43]. UGT1A1-deficient patients are homozygous carriers of the $* 28$ variant (seven TA repeats, versus six repeats in $1 * 1$ ). In addition, the germline variant-toxicity relationship has been validated between dihydropyrimidine dehydrogenase (DPD) and fluoropyrimidines. Both 5-fluorouracil and its oral equivalent capecitabine are routinely used in the treatment of gastroesophageal, hepatocellular and colorectal cancers. DPD is responsible for up to $85 \%$ of the catabolism of fluoropyrimidine antimetabolites [29], and the $D P Y D^{*} 2 A$ variant has been associated with decreased DPD catabolic activity.

The germline variants described above are associated with increased risk of myelotoxicity (most notably neutropenia), as well as other potentially lethal clinical sequelae. Myelosuppression is often the DLT of many chemotherapeutic agents due to their mechanisms of action, which target highly replicating cells such as those of the bone marrow. Neutropenia can be routinely measured and quantified, which increases the ability to characterize pharmacodynamic effects of these medications with relative precision. Complications associated with neutropenia (i.e., infection and sepsis) are associated with poorer outcomes and increased costs to the health system. It has been estimated that the cost of inpatient treatment of neutropenia for one patient exceeds US $\$ 13,000$ per hospitalization [44]. Overall, severe neutropenia has a detrimental effect on dose intensity, not only in terms of the drug causing neutropenia but also to all other combined chemotherapies administered as part of that regimen $[19,45]$. TPMT-deficient patients are at greater risk for 6mercaptopurine-induced ADRs, mainly that of severe myelosuppression [37,38]. The positive predictive value of the TPMT genotype test has been estimated to be 67-100\% [46]. UGT1A1-deficient patients are at a 9.3-fold higher risk for the development of irinotecaninduced grade 4 neutropenia than patients with nondeficient UGT1A1 activity [47]. The positive predictive value of a $U G T A 1 A 1 * 28 * 28$ genotype to detect grade 3-4 toxicity has been estimated to be 50\% [48]. Approximately $20-50 \%$ of patients who experience a grade 3-4 ADR are carriers of $D P Y D^{*} 2 A$ [49-52], and exhibit decreased DPD activity [52]. They have a 3.4-fold higher risk for grade 4 neutropenia when compared with patients with normal DPD activity [53]. The positive predictive value of $D P Y D^{*} 2 A$ for grade 3-4 toxicity has been estimated at $46 \%$ [54].

Germline variants have also been associated with chemotherapy-induced ADRs other than myelotoxicities, such as neutropenia. Genetic associations have been investigated linking 
hypertension with bevacizumab and skin toxicities with EGFR inhibitors (i.e., cetuximab, panitumumab, erlotinib and gefitinib). Skin rash has been reported in approximately $80 \%$ of patients taking these drugs [55]. Inhibition of EGFR signaling in basal keratinocytes leads to abnormalities in cell growth, differentiation and maturation, as well as an inflammatory response, which ultimately leads to the onset of rash [56]. One proposed germline polymorphism associated with skin rash is a CA dinucleotide repeat in intron 1 of $E G F R$ [57]. The number of CA repeats is inversely proportional to expression of $E G F R$, and shorter CA repeats have been linked to greater incidence of skin toxicity [58-60]. Bevacizumab is a monoclonal antibody that binds to VEGF and inhibits angiogenesis. Evidence suggests a link between hypertension and impaired angiogenesis [61], and treatment with bevacizumab has been associated with increased incidence of grade 3-4 hypertension $[16,62,63]$. A meta-analysis of 12,656 patients demonstrated that bevacizumab significantly increases the risk of all-grade hypertension by $23.6 \%$ (95\% CI: 20.5-27.1\%) and grade 3-4 hypertension by $7.9 \%$ (95\% CI: $6.1-10.2 \%$ ), relative to control patients on concurrent traditional chemotherapies [63]. In another study of patients who took bevacizumab, two VEGF germline genotypes (-634CC and -1498TT) were associated with lower rates of grade 3-4 hypertension [64]. This was the first trial to investigate biomarkers associated with bevacizumab-induced hypertension. Emerging data have confirmed a similar relationship and showed that patients with either the -634CC or -1498TT genotypes who were treated with the VEGF-pathway inhibitor sunitinib had less incidence of hypertension ( $\mathrm{p}=0.03$, in both cases) [65]. Nevertheless, at this stage, the variants in EGFR and VEGF require additional validation before they can be used as predictive markers of toxicity.

The process of pharmacogenetic marker validation still faces several major hurdles before these markers are integrated into routine clinical practice. Conflicting data generated from pharmacogenetic trials have been reported for many of the most well-characterized potential markers. For example, the $U G T 1 A 1^{*} 28 * 28$ genotype was not associated with severe diarrhea (another common side effect of irinotecan) in one study, but it was in others $[47,66,67]$. In addition, inconsistencies concerning the functional variant associated with a particular ADR complicate the validation of pharmacogenetic markers. For example, data points to -216G/T and -191C/A EGFR variants, rather than C A dinucleotide repeats in intron 1, as the causative single nucleotide polymorphisms responsible for EGFR inhibitorinduced skin toxicity [57]. The relationship between 5-fluorouracil toxicity and $D P Y D^{*} 2 A$ also illustrates another problem in the process of pharmacogenetic marker validation: rare germline variants, which have limited predictive power. Data suggests that alternate germline variants in $D P Y D$ [68], noncoding region genomic variants found through haplotype assessment [69], DPYD promoter hypermethylation [70-72], or the influences of $T Y M S, M T H F R$ and other genes [29,33], may play a significant role in explaining the remainder of the germline variant-toxicity relationship involving neutropenia and 5fluorouracil.

\section{Effect of the germline variant-toxicity relationship on chemotherapy dosing}

While over 20 chemotherapeutic agents have references to pharmacogenetic markers in their FDA label [202], a clear disconnect exists between these pharmacogenetic markers and dosing recommendations [73]. Only 6-mercaptopurine, 6-thioguanine, nilotinib and irinotecan include pharmacogenetic-based dose-reduction recommendations in their drug labels [73]. However, given the limited scale of clinical investigations on genotype-based dose individualization, they did not recommend mandated pharmacogenetic testing, nor did they provide guidance concerning the optimal timing for testing. This same limited scale of clinical investigations led the FDA to exclude explicit dose-reduction guidelines from the irinotecan or 6-mercaptopurine labels [73]. While the package insert for irinotecan includes language endorsing dose reductions by one level for patients with the UGT1A1*28*28 
genotype, these recommendations are not sufficiently specific [74]. Genotype-based dosemodification information should be far more precise as vague dose reduction language could conceivably compromise the efficacy of irinotecan if clinicians reduce doses too much.

Despite the fact that the 6-mercaptopurine label does not contain specific dose reductions based on genotype, accumulating data regarding its dosing in TPMT-deficient heterozygous [75] and homozygous [76] patients led to guidelines supporting 50\% and at least $90 \%$ reductions, respectively, of the initial dose of 6-mercaptopurine in pediatric patients treated for ALL. This genotype-based dosing strategy has successfully limited ADRs [77]. These conclusions were based on studies showing that TPMT-deficient heterozygotes can tolerate $65 \%$ of the standard $75 \mathrm{mg} / \mathrm{m}^{2} 6$-mercaptopurine dose used in pediatric ALL $\left(50 \mathrm{mg} / \mathrm{m}^{2}\right.$ daily) $[76,78,79]$, while homozygotes can receive one tenth to one fifteenth of $t$ he standard dose (i.e., $10 \mathrm{mg} / \mathrm{m}^{2}$ every third day) [75]. In subsequent studies, patients were prospectively screened for TPMT status, and doses of 6-mercaptopurine were reduced in patients with $T P M T$-deficient alleles, according to a prespecified dosing algorithm [80,81]. As a result, these patients were successfully treated with 6-mercaptopurine, with rates of grade 3-4

ADRs comparable with patients with wild-type TPMT [77]. In addition, the identification of TPMT-deficient patients not only permits clinicians to rationally reduce doses of 6mercaptopurine to avoid severe ADRs, it also allows them to preserve the dose intensity of the regimen by administering concurrent chemotherapies at unadjusted doses and without interruptions $[82,83]$.

The irinotecan doses used in current regimens were previously identified through Phase I development without stratifying patients for $U G T 1 A 1 * 28$. It has been postulated that the dose of irinotecan might be suboptimal in patients with $* 1 * 28$ and $* 1 * 1$ genotypes, and that these patients may benefit from higher, safe doses. This hypothesis has been tested in colorectal cancer patients treated with irinotecan combined with 5-fluorouracil (folinic acid, fluorouracil and irinotecan regimen) [84]. Dose-escalation of irinotecan was assessed in *1/ $* 1$ and $* 1$ * 28 patients, and results from these studies showed they were safely and effectively treated with irinotecan doses of 370 and $310 \mathrm{mg} / \mathrm{m}^{2}$, respectively. These doses were considerably higher than the $180 \mathrm{mg} / \mathrm{m}^{2}$ dose currently used in the clinic. Similar dose levels were safely given in another study of folinic acid, fluorouracil and irinotecan regimen (390 and $340 \mathrm{mg} / \mathrm{m}^{2}$, respectively) [85]. This study also demonstrated that the MTD for *28/ * 28 patients was $130 \mathrm{mg} / \mathrm{m}^{2}$, which is $30 \%$ lower than the standard dose. A similar conclusion has been also obtained in Japanese patients with $* 28$ and/or $* 6$ alleles, the common deficient alleles in Asians. Patients with $* 28 * 28 * 28 * 6$ and $* 6 * 6$ also achieved an MTD of $150 \mathrm{mg} / \mathrm{m}^{2}$ [86]. Meta-analyses demonstrated that $* 28 / 28$ patients have a significantly higher risk for grade 3-4 neutropenia when compared with *1*28 and *1*1 patients at low doses (relative risk $=2.43 ; \mathrm{p}=0.003$ ) [87], medium doses (odds ratio [OR] = $3.22 ; \mathrm{p}=0.008)$ and high doses $(\mathrm{OR}=27.8 ; \mathrm{p}=0.005)$ of irinotecan [88].

There are additional examples where a germline variant-toxicity relationship might alter dosing. In a capecitabine study of 568 patients with advanced colorectal cancer, all of the patients with $D P Y D^{*} 2 A$ experienced at least one grade 3-4 ADR (mostly diarrhea) [89]. The cumulative percentage dose reduction in these patients was significantly higher (50\%) than wild-type patients (10\%). For EGFR and VEGF-pathway inhibitors, there are no data on the effect of variants for skin rash and hypertension (respectively) on dosing and dose intensity. This represents a significant gap in current knowledge that should be addressed.

\section{The downstream effect of germline variants of toxicity on dosing \& efficacy}

The assumption that patients should be treated at the highest, safe doses of chemotherapy is still the foundation of a successful treatment regimen. Although we believe a 
pharmacogenetic marker of toxicity has high clinical utility, translation of such markers to clinical practice will be increased dramatically if ADRs can be linked to efficacy and survival. Currently, only the example of TPMT and 6-mercaptopurine exists where the knowledge of a germline variant-toxicity-dosing relationship is a tool to optimize efficacy. When 6-mercaptopurine doses were determined prospectively, based on TPMT genetic status, relapse rates were not significantly different between wild-type patients and those with TPMT-deficient genotypes $(13.2 \pm 2.3 \%$ vs $6.7 \pm 6.7 \% ; p=0.46)$. In addition, patients with TPMT-deficient genotypes were not at significantly higher rates of grade 3-4 neutropenia when compared with wild-type patients $(\mathrm{OR}=1.4 ; 95 \% \mathrm{CI}: 0.3-6.9 ; \mathrm{p}=0.71$ ) [77,79]. Most importantly, this genotype-based dosing strategy has successfully limited potentially life-threatening ADRs, while not sacrificing efficacy in this patient population $[77,82]$. The interaction between 6-mercaptopurine and the bone marrow is unique as it is not only activated intracellularly in the bone marrow, but is also the site for its antileukemic efficacy and its myelotoxic DLT. This idiosyncrasy of 6-mercaptopurine allows the downstream effects of TPMT genetics on survival to be more directly assessable. But aside from the TPMT example, the relationship between germline variant-toxicity, chemotherapy dosing and survival outcomes have been extremely difficult to establish. For other relationships, the consequences on survival are tested as a secondary end point or have not been tested at all in clinical trials.

Very few genotype-driven dose-optimization studies have prospectively assessed objective response rate, progression-free survival, overall survival or other measures of efficacy as their primary end points. For example, little evidence exists to conclusively link UGT1A1 germline variants to ADRs, dose and survival. In a retrospective analysis, dose reductions of irinotecan due to $U G T 1 A 1^{*} 28$-related toxicity did not affect progression-free survival (10 vs 11 months) or overall survival (19 vs 18 months) [90]. A second study retrospectively evaluated the association between UGT1A1 genotype, prevalence of grade 3-4 toxicity and survival outcomes in colorectal cancer patients treated with irinotecan. This study demonstrated a significantly higher rate of ADRs, coupled with worse survival, for the *28/ $* 28$ patients. Grade $3-4$ neutropenia was significantly more common in $* 28 / * 28$ patients ( $24 \%$ compared with 8.2 and $5.5 \%$ in the $* 1 / * 28$ and $* 1 / * 1$ genotypes, respectively; $\mathrm{p}=$ $0.019)$, while the median overall survival for the three genotypes was also significantly different $(2.4,2.0$ and 1.6 years for the $* 1 / * 1, * 1 / * 28$ and $* 28 / * 28$ genotypes, respectively; $\mathrm{p}$ $=0.008$ ) [91]. It is currently unclear whether the results of this study point to a true downstream effect of the $* 28 / * 28$ genotype-neutropenia relationship on survival, or if the decrease in median overall survival for patients with the $* 28 / * 28$ genotype is simply due to neutropenia-induced dose modifications that decrease overall exposure to irinotecan. Two recent studies of irinotecan, dose-escalated by genotype, showed that patients receiving higher doses experienced higher response rates than those treated at lower doses. In the first study, $65 \%$ of the patients treated with doses greater than or equal to the MTD of irinotecan achieved a complete or partial response compared with only $25 \%$ of patients treated with doses below the MTD of irinotecan $(\mathrm{p}=0.014)$. Irinotecan dosed above MTD was an independent predictor of response in these patients $(\mathrm{OR}=4.38$; 95\% CI: $1.13-17.03 ; \mathrm{p}=$ $0.03)$. Median time to progression was not significantly different between the patients treated above and below the irinotecan MTD (hazard ratio $=0.85 ; 95 \%$ CI: $0.40-1.80$ ) [84]. In the second study, $67 \%$ of patients treated with irinotecan doses $2260 \mathrm{mg} / \mathrm{m}^{2}$ achieved a complete or total response, compared with only $24 \%$ of patients treated with doses $<260 \mathrm{mg}$ / $\mathrm{m}^{2}(\mathrm{p}=0.001)$. However, unlike the previous study, the median time to progression was higher in patients treated with doses $\geq 260 \mathrm{mg} / \mathrm{m}^{2}$ when compared with patients treated with $<260 \mathrm{mg} / \mathrm{m}^{2}$ (16 vs 7 months; $\mathrm{p}=0.003$ ) [85]. These data are premature, and prospective intervention studies should test the superiority of genotype-driven dosing of irinotecan versus standard dosing in relation to survival in metastatic colorectal cancer. 
The evidence is still too sparse to provide a complete understanding of the relationship between germline variants associated with ADRs and survival in patients treated with EGFR and VEGF-pathway inhibitors, largely due to a lack of validated single nucleotide polymorphisms predictive of toxicity. Several Phase II-III studies have linked the occurrence and severity of EGFR inhibitor-induced rash to improvements in survival [22$25,92]$. One notable Phase III trial of NSCLC patients revealed a substantial survival benefit when patients experienced grade 2 and 3 rash compared with when no rash was observed (7 and 11 months, respectively, vs 3 months) [22]. Moreover, several studies have reported an association between higher response and survival with fewer CA repeats (independent from the effects of skin rash or other toxicities) [57,93], while other studies have failed to validate this relationship [94]. A prospective Phase II dose-driven erlotinib study in NSCLC patients attempted to discover whether dose escalation beyond MTD would confer a survival advantage. The results from this dose-to-rash erlotinib dosing study revealed that all patients who achieved at least a partial response also experienced rash. Progression-free survival was significantly extended in patients who experienced grade 2 rash when compared with patients without rash (3.5 vs 1.9 months; hazard ratio $=0.52 ; \mathrm{p}=0.05)$ [26]. However, this study did not investigate the role of germline variants on incidence and severity of rash, dose escalation or survival. Furthermore, the effects of dose reductions of EGFR inhibitors, as a result of grade 3-4 rash, on efficacy and survival have not been assessed. Clearly in the case of EGFR inhibitors, the germline variant-rash-dose-escalation (or reduction) relationship and its effects on efficacy and survival require further and deeper examination.

Studies of patient populations have linked the incidence of bevacizumab-induced grade $3-4$ hypertension to improved survival outcomes [27,28]. More recently, associations between $V E G F$ variants and survival have been explored $[95,96]$. A trial of advanced breast cancer patients treated with bevacizumab tested VEGF germline variants (-634G/C, -1498T/C, $-2578 \mathrm{C} / \mathrm{A}$ and $-1154 \mathrm{G} / \mathrm{A}$ ) for associations with hypertension and survival. This study demonstrated that patients with the $-634 \mathrm{CC}$ and 1498TT genotypes who experienced grade 3-4 hypertension achieved superior median overall survival compared with patients with no hypertension ( 38.7 vs 25.3 months; $\mathrm{p}=0.002$ ), while the $-634 \mathrm{G} / \mathrm{C}$ and $-1498 \mathrm{C} / \mathrm{T}$ variants were not associated with improved survival [64]. However, the results of these associations have not been readily replicated, and data from other studies have associated improved survival outcomes with the -634GG [96] and 1498CC genotypes [97]. However, data from this trial did reveal the potentially important impact of the -2578AA and -1154AA genotypes on improved survival outcomes [64]. The -2578A and the -1498T alleles are in high linkage disequilibrium in the 1000 Genomes reference population of Caucasians (Linkage disequilibrium: $\mathrm{r}^{2}=0.966$ ) [98]. This suggests that a linkage disequilibrium block of genetic variation containing these variants may impact both phenotypes, and could potentially be useful as predictive markers of both hypertension and survival.

High VEGF expression and microvascular density have been associated with decreased survival [99], and the results from the previous study showed that the -2578A allele trended toward lower VEGF expression [64]. A plausible mechanistic explanation is that the -2578AA genotype causes lower VEGF expression and that lower levels of VEGF may slow angiogenesis and the vascularization of the tumor, therefore contributing to increased survival. A similar process may also account for bevacizumab-induced hypertension. It can be postulated that inhibition of the VEGF pathway, as a result of bevacizumab administration and in combination with the -1498TT and -634CC genotypes, may result in impaired vascularization. Lower VEGF levels leads to rarefaction (a process that results in reduced microvascular density), increased peripheral vascular resistance and ultimately the onset of hypertension [61]. The impact of these germline variants, in terms of their effects on hypertension and survival, has just begun to be analyzed in studies of other VEGFpathway inhibitors, including sunitinib [65]. Such studies could be extremely useful to 
prioritize validation of these variants in patients prescribed other targeted therapies from this class, which all share similar rates of high-grade hypertension. These findings exemplify the challenges facing researchers and clinicians, and illustrate the difficulty in applying such information to guide dosing. Although we have started to understand the intricacy of the effects of $V E G F$ genetic variation on both hypertension and survival, more efforts should address whether genotype-driven hypertension of bevacizumab or sunitinib can negatively impact dosing and compromise survival.

\section{Conclusion}

Incorporation of validated pharmacogenetic markers into routine practice is of paramount importance to clinicians as they strive to reduce severe and life-threatening ADRs. Prevention and mitigation of ADRs improves patient quality of life and compliance to chemotherapy, which ultimately may lead to better survival outcomes. Understanding the putative relationship between germline variants, ADRs, chemotherapy dosing and survival is essential to the identification and translation of pharmacogenetic markers. We believe that any validation approach of pharmacogenetic markers, with potential clinical utility, must reflect incorporation of these four key components.

The examples of TPMT and 6-mercaptopurine, UGT1A1*28 and irinotecan, DPYD and the fluoropyrimidines, EGFR and EGFR inhibitors, and $V E G F$ and VEGF-pathway inhibitors provide illustrations of the varying levels of success that researchers and clinicians have had in translating pharmacogenetic molecular markers to the clinic. Currently, the example of prospective TPMT screening and subsequent dose adjustments in pediatric ALL patients has been the model for pharmacogenetic success in oncology. Increased numbers of prospective clinical trials, similar to those used to justify prospective TPMT testing, are direly needed to validate many more pharmacogenetic markers. Moreover, specific dosing recommendations need to be included into drug labels to help guide clinician decision-making processes. Dosing recommendations based on TPMT and UGT1A1 genotypes should be clarified and prospective trials should be conducted to solidify the relationship linking $D P Y D, E G F R$ and $V E G F$ variants to ADRs.

While a few smaller trials have explored the effect of genotype on ADRs, dosing and survival, only a handful of studies have conducted well-powered prospective trials to explore this relationship. This could be due to a number of reasons, ranging from low frequency of a particular germline variant (i.e., $D P Y D^{*}$ 2), to a lack of a convincing body of evidence conclusively linking a germline variant to ADRs and dose modifications (i.e., EGFR- and VEGF-pathway inhibitor germline variants). The DPYD example illustrates how the current approach of trying to identify highly penetrant, single gene variants to explain complex phenotypes (such as ADRs) and to be used as pharmacogenetic markers, may be partially flawed. Research to identify novel markers may rely on more sophisticated methods to characterize polygenic effects. In addition, research to identify translatable pharmacogenetic makers with downstream effects on survival may be complicated by the inability to take into account the effects of somatic mutations, tumor heterogeneity, epigenetic factors or other undiscovered prognostic confounders. However, identification of pharmacogenetic markers, validated through approaches that consider the germline varianttoxicity-survival relationship, will help clinicians craft strategies that rationally dose drugs within an enlarged therapeutic window to optimize efficacy while mitigating ADRs.

\section{Future perspectives}

Adoption of validated pharmacogenetic markers into routine clinical practice has been slow, in part due to the scarcity of genotype-driven trials that implement the approach we have 
outlined. As the field of pharmacogenetics continues to evolve, retrospective meta-analyses of promising gene variants might validate or refute their use in the clinic. Prospective interventional studies involving patients stratified by genotype will be a means of systematically collecting phenotype data, while also collecting data on potential confounding factors that would inhibit translation of germline variants into clinically useful pharmacogenetic markers. Finally, secondary pharmacogenetically-driven posthoc analyses of Phase III studies (which are sufficiently powered for genomic analysis) will further analyze the relationship between genetics, dosing strategies, ADRs and survival outcomes. Candidate gene and genome-wide association studies are two methods that will continue to drive identification of pharmacogenetic markers [100]. As the number of approved oral chemotherapeutic medications increases, strategies based on germline variant-toxicity-dose paradigms need to be developed to help identify patients at increased risk for the onset of common ADRs that adversely affect patient quality of life and compliance to therapy.

Existing statistical models might aid in the process of elucidating the links that connect germline variants to toxicity, dosing and survival. These relationships will be better understood by evaluating a cumulative incidence of an event in the context of a regression model of competing risks. Interdependence of variables limits the ability of existing statistical models to extricate one event of interest, for analysis, from all other competing risks. Frequently in cancer studies, several competing events (phenotypes) are present that cause treatment failures. The event of interest might occur later in treatment than the occurrence of severe ADRs, disease progression or death, which could result in early withdrawal from the study. Use of a standard case-control approach for analyzing the data might be flawed as competing risks and cumulative incidences are ignored, and has the potential to shroud the identification of pharmacogenetic markers. In recent years, cancer researchers have successfully implemented novel methodologies [101,102] into cumulative incidence competing risks models in their analyses [28,103-105]. Future studies that analyze pharmacogenetic markers, should employ these types of analyses because the intricacies of the model allow for a more accurate depiction of probabilities of events of interest occurring in the presence of competing risks. By employing this statistical model, researchers will be able to compare cumulative incidence curves between two or more groups, stratified by genotype, for a phenotype of interest.

Another reason for the slow inclusion of pharmacogenetic biomarkers into clinic is the conspicuous absence of explicit pharmacogenetic-based dosing guidelines published by regulatory bodies. The Clinical Pharmacogenetics Implementation Consortium (CPIC) of the NIH's Pharmacogenomics Research Network [203] and the Pharmacogenomics Knowledge Base [204] provide peer-reviewed, updated, evidence-based, freely accessible pharmacogenetic guidelines for chemotherapeutic agents [84]. These guidelines will facilitate the translation of knowledge derived from laboratory-based pharmacogenetic and pharmacogenomic research into the clinic, and provide explicit instructions regarding dosing and testing of these agents. Dosing information will be customized for patients based on treatment response. Guidelines have already been developed for TPMT and 6-thioguanine and 6-mercaptopurine dosing [85], but the CPIC and other consortia will continue to develop guidelines for gene-chemotherapy pairings into the future. In addition to CPIC, other consortia, such as the Pharmacogenetics Working Group from the Netherlands, will also continue to publish pharmacogenetic-based dosing guidelines for chemotherapy and targeted agents. To date, the Pharmacogenetics Working Group has published dosing guidelines for tamoxifen, irinotecan, 6-mercaptopurine and 5-fluorouracil/capecitabine, based on pharmacogenetic markers, but will continue to publish guidelines that will complement the work of the CPIC and PharmGKB [106]. Published pharmacogenetic-based dosing guidelines will undoubtedly aid clinicians in the optimization of treatment efficacy, 
mitigation of toxicity, and improvement of both medication adherence as well as quality of life for patients taking chemotherapy.

Finally, interethnic differences in the distribution of variant alleles could also potentially obstruct the adoption of pharmacogenomic standards and impede adoption of pharmacogenetic biomarkers into the clinic. Projects, such as the Pharmacogenomics for Every Nation Initiative [205], will serve to address interethnic differences in allele frequency distributions. Currently, the Pharmacogenomics for Every Nation Initiative has established regional centers in six countries, and is determining allele frequencies of known polymorphisms in different populations. Ultimately, initiatives like the Pharmacogenomics for Every Nation Initiative will help identify interethnic differences that aid in medication formulary decisions, population stratification in genotype-driven clinical trials, and even target identification early-on in the global drug development process.

\section{Acknowledgments}

The authors would like to thank D Glubb for his ideas, input, criticisms and critical review during the formulation, research and authorship of this manuscript. The authors would also like to thank A Etheridge, M Gibeault and D Schlafer for their help in the review and preparation of this manuscript.

\section{References}

Papers of special note have been highlighted as:

- of interest

II of considerable interest

1. Hoskins JM, Carey LA, Mcleod HL. CYP2D6 and tamoxifen: DNA matters in breast cancer. Nat Rev Cancer. 2009; 9(8):576-586. [PubMed: 19629072]

2. Evans WE, Relling MV. Moving towards individualized medicine with pharmacogenomics. Nature. 2004; 429(6990):464-468. [PubMed: 15164072]

3. Evans WE, Mcleod HL. Pharmacogenomics-drug disposition, drug targets, and side effects. N Engl J Med. 2003; 348(6):538-549. [PubMed: 12571262]

4. Sassi G, Striano B, Merlo UA. A reporting system for the assessment of chemotherapy toxicity. J Oncol Pharm Pract. 2005; 11(2):63-67. [PubMed: 16460607]

5. Le Tourneau C, Lee JJ, Siu LL. Dose escalation methods in Phase I cancer clinical trials. J Natl Cancer Inst. 2009; 101(10):708-720. [PubMed: 19436029]

6. Davies EC, Green CF, Taylor S, Williamson PR, Mottram DR, Pirmohamed M. Adverse drug reactions in hospital in-patients: a prospective analysis of 3695 patient-episodes. PLoS ONE. 2009; 4(2):e4439. [PubMed: 19209224]

7. Bennett CL, Nebeker JR, Yarnold PR, et al. Evaluation of serious adverse drug reactions: a proactive pharmacovigilance program (RADAR) vs safety activities conducted by the Food and Drug Administration and pharmaceutical manufacturers. Arch Intern Med. 2007; 167(10):10411049. [PubMed: 17533207]

8. Toxicity of fluorouracil in patients with advanced colorectal cancer: effect of administration schedule and prognostic factors. Meta-Analysis Group in Cancer. J Clin Oncol. 1998; 16(11):35373541. No authors listed. [PubMed: 9817272]

9. Qi WX, Shen Z, Yao Y. Meta-analysis of docetaxel-based doublet versus docetaxel alone as secondline treatment for advanced non-small-cell lung cancer. Cancer Chemother Pharmacol. 2011; 69(1): 99-106. [PubMed: 21607554]

10. Jain RK, Lee JJ, Hong D, et al. Phase I Oncology studies: evidence that in the era of targeted therapies patients on lower doses do not fare worse. Clin Cancer Res. 2010; 16(4):1289-1297. [PubMed: 20145187] 
11. Sleijfer S, Wiemer E. Dose selection in Phase I studies: why we should always go for the top. J Clin Oncol. 2008; 26(10):1576-1578. [PubMed: 18332465]

12. Haines IE. Dose selection in Phase I studies: why we should always go for the most effective. J Clin Oncol. 2008; 26(21):3650-3652. [PubMed: 18640947]

13. Shaked Y, Emmenegger U, Man S, et al. Optimal biologic dose of metronomic chemotherapy regimens is associated with maximum antiangiogenic activity. Blood. 2005; 106(9):3058-3061. [PubMed: 15998832]

14. Carducci MA, Musib L, Kies MS, et al. Phase I dose escalation and pharmacokinetic study of enzastaurin, an oral protein kinase $\mathrm{C}$ beta inhibitor, in patients with advanced cancer. J Clin Oncol. 2006; 24(25):4092-4099. [PubMed: 16943527]

15. Su X, Lacouture ME, Jia Y, Wu S. Risk of high-grade skin rash in cancer patients treated with cetuximab - an antibody against epidermal growth factor receptor: systemic review and metaanalysis. Oncology. 2009; 77(2):124-133. [PubMed: 19622903]

16. Zhu X, Wu S, Dahut WL, Parikh CR. Risks of proteinuria and hypertension with bevacizumab, an antibody against vascular endothelial growth factor: systematic review and meta-analysis. Am J Kidney Dis. 2007; 49(2):186-193. [PubMed: 17261421]

17. Noens L, Van Lierde MA, De Bock R, et al. Prevalence, determinants, and outcomes of nonadherence to imatinib therapy in patients with chronic myeloid leukemia: the ADAGIO study. Blood. 2009; 113(22):5401-5411. [PubMed: 19349618]

18. Hershman DL, Shao T, Kushi LH, et al. Early discontinuation and non-adherence to adjuvant hormonal therapy are associated with increased mortality in women with breast cancer. Breast Cancer Res Treat. 2011; 126(2):529-537. [PubMed: 20803066]

19. Lyman GH. Impact of chemotherapy dose intensity on cancer patient outcomes. J Natl Compr Canc Netw. 2009; 7(1):99-108. [PubMed: 19176210]

20. Kaestner SA, Sewell GJ. Chemotherapy dosing part I: scientific basis for current practice and use of body surface area. Clin Oncol (R Coll Radiol). 2007; 19(1):23-37. [PubMed: 17305252]

21 . Foote M. The importance of planned dose of chemotherapy on time: do we need to change our clinical practice? Oncologist. 1998; 3(5):365-368. [PubMed: 10388127]

22. Wacker B, Nagrani T, Weinberg J, Witt K, Clark G, Cagnoni PJ. Correlation between development of rash and efficacy in patients treated with the epidermal growth factor receptor tyrosine kinase inhibitor erlotinib in two large Phase III studies. Clin Cancer Res. 2007; 13(13):3913-3921. [PubMed: 17606725]

23. Moore MJ, Goldstein D, Hamm J, et al. Erlotinib plus gemcitabine compared with gemcitabine alone in patients with advanced pancreatic cancer: a Phase III trial of the National Cancer Institute of Canada Clinical Trials Group. J Clin Oncol. 2007; 25(15):1960-1966. [PubMed: 17452677]

24. Giaccone G, Gallegos Ruiz M, Le Chevalier T, et al. Erlotinib for frontline treatment of advanced non-small cell lung cancer: a Phase II study. Clin Cancer Res. 2006; 12(20 Pt 1):6049-6055. [PubMed: 17062680]

25. Perez-Soler R, Chachoua A, Hammond LA, et al. Determinants of tumor response and survival with erlotinib in patients with non-small-cell lung cancer. J Clin Oncol. 2004; 22(16):3238-3247. [PubMed: 15310767]

26-. Mita AC, Papadopoulos K, De Jonge MJ, et al. Erlotinib 'dosing-to-rash': a Phase II intrapatient dose escalation and pharmacologic study of erlotinib in previously treated advanced non-small cell lung cancer. Br J Cancer. 2011; 105(7):938-944. Phase II dose-to-rash study of patients administered erlotinib, which showed that progression-free survival was significantly extended in patients who experienced at least grade 2 rash $(\mathrm{p}=0.051)$. [PubMed: 21878940]

27. Miller K, Wang M, Gralow J, et al. Paclitaxel plus bevacizumab versus paclitaxel alone for metastatic breast cancer. N Engl J Med. 2007; 357(26):2666-2676. [PubMed: 18160686]

28-. Dahlberg SE, Sandler AB, Brahmer JR, Schiller JH, Johnson DH. Clinical course of advanced non-small-cell lung cancer patients experiencing hypertension during treatment with bevacizumab in combination with carboplatin and paclitaxel on ECOG 4599. J Clin Oncol. 2010; 28(6):949-954. Original article from the ECOG 4599 study, which serves as an excellent example of the use of the cumulative incidence function and competing risks model in non-small- 
cell lung cancer patients who experienced hypertension while treated with bevacizumab, carboplatin and paclitaxel. [PubMed: 20085937]

29. Coate L, Cuffe S, Horgan A, Hung RJ, Christiani D, Liu G. Germline genetic variation, cancer outcome, and pharmacogenetics. J Clin Oncol. 2010; 28(26):4029-4037. [PubMed: 20679599]

30. Innocenti F, Schilsky RL. Translating the cancer genome into clinically useful tools and strategies. Dis Models Mech. 2009; 2(9-10):426-429.

31. Marsh S, Mcleod HL. Pharmacogenomics: from bedside to clinical practice. Hum Mol Genet. 2006; 15(1):R89-R93. [PubMed: 16651374]

32. Paugh SW, Stocco G, Mccorkle JR, Diouf B, Crews KR, Evans WE. Cancer Pharmacogenomics. Clin Pharmacol Ther. 2011; 90(3):461-466. [PubMed: 21796115]

33. Houtsma D, Guchelaar HJ, Gelderblom H. Pharmacogenetics in oncology: a promising field. Curr Pharm Des. 2010; 16(2):155-163. [PubMed: 20205661]

34. Yong WP, Innocenti F, Ratain MJ. The role of Pharmacogenetics in cancer therapeutics. Br J Clin Pharmacol. 2006; 62(1):35-46. [PubMed: 16842377]

35. Pui CH, Evans WE. Treatment of acute lymphoblastic leukemia. N Engl J Med. 2006; 354(2):166178. [PubMed: 16407512]

36. Krynetski E, Evans WE. Drug methylation in cancer therapy: lessons from the TPMT polymorphism. Oncogene. 2003; 22(47):7403-7413. [PubMed: 14576848]

37. Kishi S, Cheng C, French D, et al. Ancestry and pharmacogenetics of antileukemic drug toxicity. Blood. 2007; 109(10):4151-4157. [PubMed: 17264302]

38. Relling MV, Hancock ML, Rivera GK, et al. Mercaptopurine therapy intolerance and heterozygosity at the thiopurine S-methyltransferase gene locus. J Natl Cancer Inst. 1999; 91(23): 2001-2008. [PubMed: 10580024]

39. Beutler E, Gelbart T, Demina A. Racial variability in the UDP-glucuronosyltransferase 1 (UGT1A1) promoter: a balanced polymorphism for regulation of bilirubin metabolism? Proc Natl Acad Sci USA. 1998; 95(14):8170-8174. [PubMed: 9653159]

40. Ritter JK, Kessler FK, Thompson MT, Grove AD, Auyeung DJ, Fisher RA. Expression and inducibility of the human bilirubin UDP-glucuronosyltransferase UGT1A1 in liver and cultured primary hepatocytes: evidence for both genetic and environmental influences. Hepatology. 1999; 30(2):476-484. [PubMed: 10421657]

41. Ramirez J, Mirkov S, Zhang W, et al. Hepatocyte nuclear factor-1 alpha is associated with UGT1A1, UGT1A9 and UGT2B7 mRNA expression in human liver. Pharmacogenomics J. 2008; 8(2):152-161. [PubMed: 17440429]

42. Iyer L, Hall D, Das S, et al. Phenotype-genotype correlation of in vitro SN-38 (active metabolite of irinotecan) and bilirubin glucuronidation in human liver tissue with UGT1A1 promoter polymorphism. Clin Pharmacol Ther. 1999; 65(5):576-582. [PubMed: 10340924]

43. Iyer L, King CD, Whitington PF, et al. Genetic predisposition to the metabolism of irinotecan (CPT-11). Role of uridine diphosphate glucuronosyltransferase isoform 1A1 in the glucuronidation of its active metabolite (SN-38) in human liver microsomes. J Clin Invest. 1998; 101(4):847-854. [PubMed: 9466980]

44. Caggiano V, Weiss RV, Rickert TS, Linde-Zwirble WT. Incidence, cost, and mortality of neutropenia hospitalization associated with chemotherapy. Cancer. 2005; 103(9):1916-1924. [PubMed: 15751024]

45. Lyman GH. A comparison of international guidelines for the prevention of chemotherapy-induced neutropenia. Curr Opin Hematol. 2010 (Epub ahead of print). 10.1097/MOH.0b013e328340dc51

46. Donnan JR, Ungar WJ, Mathews M, Rahman P. Systematic review of thiopurine methyltransferase genotype and enzymatic testing strategies. Ther Drug Monit. 2011; 33(2):192-199. [PubMed: 21240057]

47. Innocenti F, Undevia SD, Iyer L, et al. Genetic variants in the UDP-glucuronosyltransferase 1A1 gene predict the risk of severe neutropenia of irinotecan. J Clin Oncol. 2004; 22(8):1382-1388. [PubMed: 15007088]

48. Innocenti F, Ratain MJ. Pharmacogenetics of irinotecan: clinical perspectives on the utility of genotyping. Pharmacogenomics. 2006; 7(8):1211-1221. [PubMed: 17184208] 
49. Watson RG, Mcleod HL. Pharmacogenomic contribution to drug response. Cancer J. 2011; 17(2): 80-88. [PubMed: 21427551]

50. Salgueiro N, Veiga I, Fragoso M, et al. Mutations in exon 14 of dihydropyrimidine dehydrogenase and 5-Fluorouracil toxicity in Portuguese colorectal cancer patients. Genet Med. 2004; 6(2):102107. [PubMed: 15017333]

51. Ahluwalia R, Freimuth R, Mcleod HL, Marsh S. Use of pyrosequencing to detect clinically relevant polymorphisms in dihydropyrimidine dehydrogenase. Clin Chem. 2003; 49(10):16611664. [PubMed: 14500593]

52. Van Kuilenburg AB, Meinsma R, Zoetekouw L, Van Gennip AH. High prevalence of the IVS14 \pm $1 \mathrm{G}>\mathrm{A}$ mutation in the dihydropyrimidine dehydrogenase gene of patients with severe 5fluorouracil-associated toxicity. Pharmacogenetics. 2002; 12(7):555-558. [PubMed: 12360106]

53. Van Kuilenburg AB, Meinsma R, Zoetekouw L, Van Gennip AH. Increased risk of grade IV neutropenia after administration of 5-fluorouracil due to a dihydropyrimidine dehydrogenase deficiency: high prevalence of the IVS14 $\pm 1 G>$ A mutation. Int J Cancer. 2002; 101(3):253-258. [PubMed: 12209976]

54. Schwab M, Zanger UM, Marx C, et al. Role of genetic and nongenetic factors for fluorouracil treatment-related severe toxicity: a prospective clinical trial by the German 5-FU Toxicity Study Group. J Clin Oncol. 2008; 26(13):2131-2138. [PubMed: 18299612]

55. Saif MW, Merikas I, Tsimboukis S, Syrigos K. Erlotinib-induced skin rash. Pathogenesis, clinical significance and management in pancreatic cancer patients. JOP. 2008; 9(3):267-274. [PubMed: 18469438]

56. Li T, Perez-Soler R. Skin toxicities associated with epidermal growth factor receptor inhibitors. Target Oncol. 2009; 4(2):107-119. [PubMed: 19452131]

57. Liu G, Gurubhagavatula S, Zhou W, et al. Epidermal growth factor receptor polymorphisms and clinical outcomes in non-small-cell lung cancer patients treated with gefitinib. Pharmacogenomics J. 2008; 8(2):129-138. [PubMed: 17375033]

58. Huang CL, Yang $\mathrm{CH}$, Yeh $\mathrm{KH}$, et al. EGFR intron 1 dinucleotide repeat polymorphism is associated with the occurrence of skin rash with gefitinib treatment. Lung Cancer. 2009; 64(3): 346-351. [PubMed: 18995924]

59. Rudin CM, Liu W, Desai A, et al. Pharmacogenomic and pharmacokinetic determinants of erlotinib toxicity. J Clin Oncol. 2008; 26(7):1119-1127. [PubMed: 18309947]

60. Amador ML, Oppenheimer D, Perea S, et al. An epidermal growth factor receptor intron 1 polymorphism mediates response to epidermal growth factor receptor inhibitors. Cancer Res. 2004; 64(24):9139-9143. [PubMed: 15604284]

61. Veronese ML, Mosenkis A, Flaherty KT, et al. Mechanisms of hypertension associated with BAY 43-9006. J Clin Oncol. 2006; 24(9):1363-1369. [PubMed: 16446323]

62. Ranpura V, Pulipati B, Chu D, Zhu X, Wu S. Increased risk of high-grade hypertension with bevacizumab in cancer patients: a meta-analysis. Am J Hypertens. 2010; 23(5):460-468. [PubMed: 20186127]

63. Jubb AM, Hurwitz HI, Bai W, et al. Impact of vascular endothelial growth factor-A expression, thrombospondin-2 expression, and microvessel density on the treatment effect of bevacizumab in metastatic colorectal cancer. J Clin Oncol. 2006; 24(2):217-227. [PubMed: 16365183]

64. Schneider BP, Wang M, Radovich M, et al. Association of vascular endothelial growth factor and vascular endothelial growth factor receptor-2 genetic polymorphisms with outcome in a trial of paclitaxel compared with paclitaxel plus bevacizumab in advanced breast cancer: ECOG 2100. J Clin Oncol. 2008; 26(28):4672-4678. Demonstarted that in a cohort of breast cancer patients treated with bevacizumab, two VEGF germline variants (-634G/C, -1498T/C) were significantly associated with hypertension, while two additional variants (-2578C/A and $-1154 \mathrm{G} / \mathrm{A})$ were significantly associated with survival. [PubMed: 18824714]

65. Kim JJ, Vaziri SA, Rini BI, et al. Association of VEGF and VEGFR2 single nucleotide polymorphisms with hypertension and clinical outcome in metastatic clear cell renal cell carcinoma patients treated with sunitinib. Cancer. 2011; 118(7):1946-1954. [PubMed: 21882181] 
66. Massacesi C, Terrazzino S, Marcucci F, et al. Uridine diphosphate glucuronosyl transferase 1A1 promoter polymorphism predicts the risk of gastrointestinal toxicity and fatigue induced by irinotecan-based chemotherapy. Cancer. 2006; 106(5):1007-1016. [PubMed: 16456808]

67. Marcuello E, Altes A, Menoyo A, Del Rio E, Gomez-Pardo M, Baiget M. UGT1A1 gene variations and irinotecan treatment in patients with metastatic colorectal cancer. Br J Cancer. 2004; 91(4):678-682. [PubMed: 15280927]

68. Kristensen MH, Pedersen PL, Melsen GV, Ellehauge J, Mejer J. Variants in the dihydropyrimidine dehydrogenase, methylenetetrahydrofolate reductase and thymidylate synthase genes predict early toxicity of 5-fluorouracil in colorectal cancer patients. J Int Med Res. 2010; 38(3):870-883. [PubMed: 20819423]

69. Amstutz U, Farese S, Aebi S, Largiader CR. Dihydropyrimidine dehydrogenase gene variation and severe 5-fluorouracil toxicity: a haplotype assessment. Pharmacogenomics. 2009; 10(6):931-944. [PubMed: 19530960]

70. Zhang X, Soong R, Wang K, et al. Suppression of $D P Y D$ expression in RKO cells via DNA methylation in the regulatory region of the $D P Y D$ promoter: a potentially important epigenetic mechanism regulating DPYD expression. Biochem Cell Biol. 2007; 85(3):337-346. [PubMed: 17612628]

71. Yu J, Mcleod HL, Ezzeldin HH, Diasio RB. Methylation of the DPYD promoter and dihydropyrimidine dehydrogenase deficiency. Clin Cancer Res. 2006; 12(12):3864. author reply 3864. [PubMed: 16778115]

72. Ezzeldin HH, Lee AM, Mattison LK, Diasio RB. Methylation of the DPYD promoter: an alternative mechanism for dihydropyrimidine dehydrogenase deficiency in cancer patients. Clin Cancer Res. 2005; 11(24 Pt 1):8699-8705. [PubMed: 16361556]

73. Maitland ML, Vasisht K, Ratain MJ. TPMT, UGT1A1 and DPYD: genotyping to ensure safer cancer therapy? Trends Pharmacol Sci. 2006; 27(8):432-437. [PubMed: 16815558]

74. Solution for intravenous injection, irinotecan hydrochloride solution for intravenous injection. Pfizer Ltd; NY, USA: 2009. CAMPTOSAR ${ }^{\circledR}$, package insert.

75. Evans WE, Horner M, Chu YQ, Kalwinsky D, Roberts WM. Altered mercaptopurine metabolism, toxic effects, and dosage requirement in a thiopurine methyltransferase-deficient child with acute lymphocytic leukemia. J Pediatr. 1991; 119(6):985-989. [PubMed: 1960624]

76. Schmiegelow K, Forestier E, Hellebostad M, et al. Long-term results of NOPHO ALL-92 and ALL-2000 studies of childhood acute lymphoblastic leukemia. Leukemia. 2010; 24(2):345-354. [PubMed: 20010622]

77. Relling MV, Pui CH, Cheng C, Evans WE. Thiopurine methyltransferase in acute lymphoblastic leukemia. Blood. 2006; 107(2):843-844. [PubMed: 16401827]

78. Schmiegelow K, Heyman M, Kristinsson J, et al. Oral methotrexate/6-mercaptopurine may be superior to a multidrug LSA2L2 maintenance therapy for higher risk childhood acute lymphoblastic leukemia: results from the NOPHO ALL-92 study. J Pediatr Hematol Oncol. 2009; 31(6):385-392. [PubMed: 19648786]

79. Stocco G, Cheok MH, Crews KR, et al. Genetic polymorphism of inosine triphosphate pyrophosphatase is a determinant of mercaptopurine metabolism and toxicity during treatment for acute lymphoblastic leukemia. Clin Pharmacol Ther. 2009; 85(2):164-172. [PubMed: 18685564]

80. Stocco G, Crews KR, Evans WE. Genetic polymorphism of inosine-triphosphate-pyrophosphatase influences mercaptopurine metabolism and toxicity during treatment of acute lymphoblastic leukemia individualized for thiopurine-S-methyl-transferase status. Expert Opin Drug Saf. 2010; 9(1):23-37. [PubMed: 20021291]

81. Pui CH, Sandlund JT, Pei D, et al. Improved outcome for children with acute lymphoblastic leukemia: results of Total Therapy Study XIIIB at St Jude Children's Research Hospital. Blood. 2004; 104(9):2690-2696. [PubMed: 15251979]

82. Relling MV, Klein TE. CPIC: Clinical Pharmacogenetics Implementation Consortium of the Pharmacogenomics Research Network. Clin Pharmacol Ther. 2011; 89(3):464-467. [PubMed: 21270786]

83m. Relling MV, Gardner EE, Sandborn WJ, et al. Clinical Pharmacogenetics Implementation Consortium guidelines for thiopurine methyltransferase genotype and thiopurine dosing. Clin 
Pharmacol Ther. 2011; 89(3):387-391. The Clinical Pharmacogenetics Implementation Consortium guidelines provide specific dosing recommendations for thiopurine agents (azathioprine, mercaptopurine and thioguanine) based on TPMT genotype. [PubMed: 21270794]

84. Toffoli G, Cecchin E, Gasparini G, et al. Genotype-driven Phase I study of irinotecan administered in combination with fluorouracil/leucovorin in patients with metastatic colorectal cancer. J Clin Oncol. 2010; 28(5):866-871. An original article where irinotecan was doseescalated by genotype and $65 \%$ of the patients treated with doses greater than or equal to the irinotecan maximum tolerated dose achieved a complete or partial response, compared with only $25 \%$ of patients treated with doses below the maximum tolerated dose of irinotecan $(p=0.014)$. [PubMed: 20038727]

85-. Marcuello E, Paez D, Pare L, et al. A genotype-directed Phase I-IV dose-finding study of irinotecan in combination with fluorouracil/leucovorin as first-line treatment in advanced colorectal cancer. Br J Cancer. 2011; 105(1):53-57. An original article where irinotecan was dose-escalated by genotype and showed $67 \%$ of patients treated with irinotecan doses $>260 \mathrm{mg} /$ $\mathrm{m}^{2}$ achieved a complete or total response, compared with only $24 \%$ of patients treated with doses $<260 \mathrm{mg} / \mathrm{m}^{2}(\mathrm{p}=0.001)$; and time to progression was higher in patients treated with doses $>260$ $\mathrm{mg} / \mathrm{m}^{2}$ compared with patients treated $<260 \mathrm{mg} / \mathrm{m}^{2}(\mathrm{p}=0.003)$. [PubMed: 21654688 ]

86. Satoh T, Ura T, Yamada Y, et al. Genotype-directed, dose-finding study of irinotecan in cancer patients with $U G T 1 A 1^{*} 28$ and/or $U G T 1 A 1^{*} 6$ polymorphisms. Cancer Sci. 2011; 102(10):18681873. [PubMed: 21740478]

87m. Hu ZY, Yu Q, Pei Q, Guo C. Dose-dependent association between $U G T 1 A 1^{*} 28$ genotype and irinotecan-induced neutropenia: low doses also increase risk. Clin Cancer Res. 2010; 16(15): 3832-3842. Demonstrated that $U G T 1 A 1 * 28 * 28$ patients have a significantly higher risk for grade $3-4$ neutropenia compared with $* 1 / * 28$ and $* 1 / * 1$ at low doses $(\mathrm{p}=0.003)$. [PubMed: 20562211]

88. Hoskins JM, Goldberg RM, Qu P, Ibrahim JG, Mcleod HL. UGT1A1*28 genotype and irinotecan-induced neutropenia: dose matters. J Natl Cancer Inst. 2007; 99(17):1290-1295. Demonstrated that $U G T 1 A 1^{*} 28 * 28$ patients have a significantly higher risk for grade $3-4$ neutropenia compared with $* 1 / * 28$ and $* 1 / * 1$ at medium doses $(\mathrm{p}=0.008)$ and high doses of irinotecan ( $\mathrm{p}=0.005)$. [PubMed: 17728214]

89. Deenen MJ, Tol J, Burylo AM, et al. Relationship between single nucleotide polymorphisms and haplotypes in DPYD and toxicity and efficacy of capecitabine in advanced colorectal cancer. Clin Cancer Res. 2011; 17(10):3455-3468. [PubMed: 21498394]

90. Liu CY, Chen PM, Chiou TJ, et al. UGT1A1*28 polymorphism predicts irinotecan-induced severe toxicities without affecting treatment outcome and survival in patients with metastatic colorectal carcinoma. Cancer. 2008; 112(9):1932-1940. [PubMed: 18300238]

91. Shulman K, Cohen I, Barnett-Griness O, et al. Clinical implications of UGT1A1*28 genotype testing in colorectal cancer patients. Cancer. 2011; 117(14):3156-3162. [PubMed: 21287524]

92. Gibson TB, Ranganathan A, Grothey A. Randomized Phase III trial results of panitumumab, a fully human anti-epidermal growth factor receptor monoclonal antibody, in metastatic colorectal cancer. Clin Colorectal Cancer. 2006; 6(1):29-31. [PubMed: 16796788]

93. Nie Q, Wang Z, Zhang GC, et al. The epidermal growth factor receptor intron 1 (CA) $n$ microsatellite polymorphism is a potential predictor of treatment outcome in patients with advanced lung cancer treated with Gefitinib. Eur J Pharmacol. 2007; 570(1-3):175-181. [PubMed: 17597605]

94. Gregorc V, Hidalgo M, Spreafico A, et al. Germline polymorphisms in EGFR and survival in patients with lung cancer receiving gefitinib. Clin Pharmacol Ther. 2008; 83(3):477-484. [PubMed: 17713473]

95. Dassoulas K, Gazouli M, Rizos S, et al. Common polymorphisms in the vascular endothelial growth factor gene and colorectal cancer development, prognosis, and survival. Mol Carcinog. 2009; 48(6):563-569. [PubMed: 19009560]

96. Kim JG, Chae YS, Sohn SK, et al. Vascular endothelial growth factor gene polymorphisms associated with prognosis for patients with colorectal cancer. Clin Cancer Res. 2008; 14(1):62-66. [PubMed: 18172253] 
97. Loupakis F, Ruzzo A, Salvatore L, et al. Retrospective exploratory analysis of VEGF polymorphisms in the prediction of benefit from first-line FOLFIRI plus bevacizumab in metastatic colorectal cancer. BMC Cancer. 2011; 11:247. [PubMed: 21669012]

98. Johnson AD, Handsaker RE, Pulit SL, Nizzari MM, O'donnell CJ, De Bakker PI. SNAP: a webbased tool for identification and annotation of proxy SNPs using HapMap. Bioinformatics. 2008; 24(24):2938-2939. [PubMed: 18974171]

99. Des Guetz G, Uzzan B, Nicolas P, et al. Microvessel density and VEGF expression are prognostic factors in colorectal cancer. Meta-analysis of the literature. Br J Cancer. 2006; 94(12):1823-1832. [PubMed: 16773076]

100. Innocenti F, Cox NJ, Dolan ME. The use of genomic information to optimize cancer chemotherapy. Semin Oncol. 2011; 38(2):186-195. [PubMed: 21421109]

101. Fine JP, Gray RJ. A proportional hazards model for the subdistribution of a competing risk. J Am Stat Assoc. 1999; 94:496-509.

102. Klein JP, Andersen PK. Regression modeling of competing risks data based on pseudovalues of the cumulative incidence function. Biometrics. 2005; 61(1):223-229. [PubMed: 15737097]

103. Lee JJ, Feng L, Reshef DS, et al. Mortality in the randomized, controlled lung intergroup trial of isotretinoin. Cancer Prev Res. 2010; 3(6):738-744.

104. Nguyen PL, Taghian AG, Katz MS, et al. Breast cancer subtype approximated by estrogen receptor, progesterone receptor, and HER-2 is associated with local and distant recurrence after breast-conserving therapy. J Clin Oncol. 2008; 26(14):2373-2378. [PubMed: 18413639]

105. Pui CH, Pei D, Sandlund JT, et al. Risk of adverse events after completion of therapy for childhood acute lymphoblastic leukemia. J Clin Oncol. 2005; 23(31):7936-7941. [PubMed: 16258093]

106". Swen JJ, Nijenhuis M, De Boer A, et al. Pharmacogenetics: from bench to byte - an update of guidelines. Clin Pharmacol Ther. 2011; 89(5):662-673. Pharmacogenetic dosing guidelines published by the Pharmacogenetics Working Group from the Netherlands. The article details the dosing recommendations for 53 drugs, including seven chemotherapeutic agents. [PubMed: 21412232]

\section{Websites}

201. National Cancer Institute. Cancer Therapy Evaluation Program NCI. Common Terminology Criteria for Adverse Events (CTCAE) v.4.03. http://evs.nci.nih.gov/ftp1/CTCAE/ CTCAE_4.03_2010-2006-14_QuickReference_8.5x11.pdf

202. US Food and Drug Administration. Table of pharmacogenomic biomarkers in drug labels. www.fda.gov/drugs/scienceresearch/researchareas/Pharmacogenetics/ucm083378.htm

203. Pharmacogenomics Research Network. www.pgrn.org

204. Pharmacogenomics The Knowledgebase. www.pharmgkb.org

205. Pharmacogenomics for Every Nation Initiative. www.pgeni.org 


\section{Executive summary}

\section{Adverse drug reactions}

- Adverse drug reactions (ADRs) account for 7\% of all hospital admissions, occur in 10-20\% of all patients and increase hospital stays.

- ADR-related morbidity exceeds US\$3.6 billion annually in the USA.

- Prevention and mitigation of ADRs improves quality of life and medication adherence, and may lead to better survival outcomes.

\section{Chemotherapy dose}

- Maintaining dose for the entire course of treatment has been shown prospectively and retrospectively in various tumor types to be correlated with better outcomes.

- Traditional cytotoxic chemotherapies are dosed to maximum tolerated dose, but novel molecularly targeted agents could be dosed to optimal biologic dose.

\section{TPMT \& 6-mercaptopurine}

- $\quad$ Reduced TPMT function places patients at significantly greater risk for 6mercaptopurine-induced ADRs, including neutropenia.

- Patients heterozygous and homozygous for reduced TPMT function alleles receive 50 and $\searrow 90 \%$ reductions of the initial dose of 6-mercaptopurine, respectively.

- $\quad$ Dose reductions in patients with reduced TPMT function results in less grade 34 neutropenia, while preserving positive survival outcomes.

\section{UGT1A1\& irinotecan}

- UGTA1A1*28*28 patients are at a significantly higher risk for grade 3-4 neutropenia at low and medium doses of irinotecan when compared with UGTA1A1*1*28 and UGTA1A1*1* 1 patients.

- $U G T 1 A 1^{*} 1$ patients were able to tolerate doses that exceed the standard irinotecan maximum tolerated dose, and dose reductions for UGT1A $1^{*} 28$ patients due to neutropenia did not compromise survival outcomes.

- Explicit dosing recommendations for patients with $U G T 1 A 1^{*} 1$ versus $U G T 1 A I^{*} 28$ have not yet been published.

\section{DPYD \& 5-fluorouracil/capecitabine}

- Germline variants in $D P Y D$ have been associated with increased ADRs.

- The $D P Y D^{*} 2$ allele is not prevalent in the general population, but is associated with a 3.4-fold higher risk for grade 4 neutropenia compared with patients with normal dihydropyrimidine dehydrogenase activity.

- The relationship between $D P Y D^{*} 2$ genotype, grade 3-4 neutropenia and survival remains unclear and requires further investigation.

\section{EGF receptor \& erlotinib/cetuximab}

- Skin rash is an ADR that is experienced by approximately $80 \%$ of patients taking EGF receptor (EGFR) inhibitors. 
- A CA repeat in the intron 1 region of $E G F R$ has been associated with increased incidence of rash in patients taking EGFR inhibitors.

- Increased incidence of rash has been associated with increased progression-free survival.

- The role of germline variants in EGFR inhibitor-induced rash, dosing and survival remains unclear.

\section{VEGF \& bevacizumab}

- Correlations between bevacizumab-induced hypertension and improved survival outcomes may be related to germline variations in the VEGF pathway.

- VEGF germline variants have been associated with decreased incidence of hypertension (-634CC and -1498TT) and increased survival (-2578AA and -1154AA).

- The relationship between VEGF-pathway germline variants and bevacizumab dosing is still unclear.

\section{Conclusion}

- Any approach to clinical validation of pharmacogenetic markers must reflect an understanding of the relationships between germline variants, ADRs, chemotherapy dosing and survival.

- Consortia, such as the Pharmacogenomics Knowledge Base, the Clinical Pharmacogenetics Implementation Consortium and the Pharmacogenetics Working Group will provide pharmacogenetically-based dosing recommendations for chemotherapeutics and aid in the translation of pharmacogenetic markers into clinical practice. 